\title{
Editorial: Hysteresis Characterization and Control of Electrorheological and Magnetorheological Materials
}

\author{
Xian-Xu Frank Bai $^{1 *}$, Ramin Sedaghati ${ }^{2}$, Janusz Goldasz ${ }^{3}$ and Shuaishuai Sun ${ }^{4}$ \\ ${ }^{1}$ Laboratory for Adaptive Structures and Intelligent Systems (LASIS), Department of Vehicle Engineering, Hefei University of \\ Technology, Hefei, China, ${ }^{2}$ Department of Mechanical and Industrial Engineering, Concordia University, Montreal, QC, Canada, \\ ${ }^{3}$ Faculty of Electrical and Computer Engineering, Cracow University of Technology, Kraków, Poland, ${ }^{4}$ Department of Precision \\ Machinery and Instrumentation, University of Science and Technology of China, Hefei, China
}

Keywords: hysteresis, magnetorhelogical, electrorheological, smart material, inverse problem, vibration control

Editorial on the Research Topic

Hysteresis Characterization and Control of Electrorheological and Magnetorheological Materials

\section{INTRODUCTION}

Smart materials, specific referring to electrorheological/magnetorheological (MR) fluids and elastomers, are providing incomparable prospect of the "old" applications, such as vehicular/seat suspensions with vibration/shock mitigation control, engine mount, brake and torque transmission systems, anti-earthquake structures for civil buildings, medical rehabilitation actuators, robotic end effectors, active vales/actuators. However, from academic and industrial viewpoints, hysteresis properties of the materials and the structures are the core of the obstacle of "optimal" applications.

Different with many other well-established smart materials and structures journals such as Smart Materials and Structures and Journal of Intelligent Material Systems and Structures, the primary objective of this Special Issue in Frontiers in Materials is to provide an open-access forum for researchers and practitioners to exchange their latest achievements and to identify critical issues and challenges for future investigation on the hysteresis-based design, modelling, optimization and control dynamic systems. This special issue has accepted 13 high-quality papers after rigorous interactive peer-review processes, the contents of which are summarized as follows.

Specialty section:

This article was submitted to Smart Materials, a section of the journal

Frontiers in Materials

Received: 28 June 2021 Accepted: 05 July 2021 Published: 16 July 2021

Citation:

Bai X-XF, Sedaghati R, Goldasz J and Sun S (2021) Editorial: Hysteresis Characterization and Control of Electrorheological and Magnetorheological Materials.

Front. Mater. 8:732353

doi: 10.3389/fmats.2021.732353

\section{HYSTERESIS IN MATERIALS}

Sokolovski et al. enhanced the MR effect with the shear-thickening (ST) property. The viscosity of ST fluids when subjected to sudden impacts increases dramatically which may provide a viable alternative to typical MR suspensions in certain applications. The authors measured properties of MRST materials with different iron concentrations, thus demonstrating behaviours of the material, i.e., from a material with a weak MR effect and a strong ST property to the one with a strong MR effect and less pronounced ST behaviour. The authors classified the MRST fluid behaviour into three regimes for designing application-oriented materials.

In another study involving MRST fluids Yu et al. demonstrated the performance of an MR damper using such material for seismic applications. Moreover, the damper includes a fail-safe circuit (in the form of a permanent magnet) for bi-directional control. The authors presented a working principle of the damper, show their finite-element calculations of the magnetic field 
distribution in the MR valve, and then highlighted the experimental results of the damper subjected to various excitation inputs. It is shown that the device has the ability to be controlled by magnetic field, and reveal the ST behaviour at the same time.

In the work by Zhang et al., the dynamic mechanical properties of MR elastomers under cyclic loadings and periodic magnetic field were investigated and analyzed, including the influences of matrix, particle distribution, magnetic field on the dynamic mechanical hysteresis. They found that all the mechanical pressure, shear strain, as well as periodic magnetic field would cause a hysteresis in the dynamic mechanical properties of the MR elastomer, and the hysteresis tends to be saturated after cycles.

\section{HYSTERESIS IN DEVICES}

Lozoya-Santos et al. presented a general modeling approach for MR shock absorbers using the characteristic diagrams, i.e., forcedisplacement, force-velocity, and force-acceleration loops. One certain pattern of seven can be obtained for model design after analyzing the diagrams at different frequencies of interest. The experimentally validated new general modeling approach with simply mathematical expression could provide decent performance with $2-10 \%$ errors.

Gołdasz et al. thoroughly investigated the hysteretic behavior of MR dampers. Different with the existing models for combined hysteretic behavior of MR actuators by observing the relationships between the output and the inputs. They presented and explained that the two hysteretic mechanisms mechanical/hydraulic and magnetic are the distinct terms, and they should be split in the modeling process. Both the Duhem model and the derived Maxwell type model were used in the work for illustration purposes. Based on the approach they proposed, each hysteresis mechanism can be studied independently, and then essential understanding of the hysteretic behavior of MR devices can be obtained.

Guo et al. investigated the nonlinear behavior of MR damper with emphasis on the coupled inertial-viscous-frictional-elastic transients of the two-dimensional (2D) axisymmetric flow of the compressible Herschel-Bulkley fluid. The viscoplasticity and compressibility of MR fluid are, respectively, modeled by the modified Herschel-Bulkley model and the Tait equation. Significant conclusions were: coupled transients of an MR damper are frequency dependent, and the weak compressibility of MR fluid mainly happens in the chamber rather than in the working gap and is crucial to accurately predict and understand the dynamic performances of MR dampers. It is of help in research on general MR devices design and modeling.

In the work by $\mathrm{Li}$ et al., they attempted to reduce or eliminate the effect of the magnetic hysteresis of the MR damper using JilesAtherton (J-A) model and a magnetic hysteresis compensation control method was proposed. Magnetic induction intensity was obtained via an embedded Hall sensor in MR fluid damper. The proposed hysteresis compensation method with a PID controller can eliminate the effect of hysteresis under both low frequency and high frequency inputs. The output damping force of the MR impact buffer system indicated that the buffer performance has been improved by employing the magnetic hysteresis compensation control method.

Zhong et al. combined a MR damper and a MR inerter according to the design concept of "functional integration", and realized an integrated shock absorber with both tunable inertance and damping. A new integrated inerter-spring-damper with both adjustable inertance and damping characteristics when applied with coil currents was further proposed. Mechanical output characteristics of the integrated inerter-spring-damper were analyzed, and the integrated inerter-spring-damper-based $1 / 4$ vehicle suspension provided an improved vibration mitigation performance.

\section{HYSTERESIS IN SYSTEMS}

In the applications of seat suspensions/isolation systems, Zhu et al. and Liu et al. studied a cab seat suspension with a MR fluid damperand a MR elastomer-based semi-active seat suspension isolator, respectively. In order to address the core problem of the nonlinear hysteresis, Zhu et al. presented a unified-format model expression for both Bingham and Bouc-Wen models, while Liu et al. used the adaptive neural network. Experimental results of the two semi-active systems show the superiority of the controlled systems.

Specifically, for the system controller of another application of MR semi-active seat suspension, Liu et al. proposed a new controller - particle swarm optimization (PSO)-improved fruit fly optimization algorithm (IFOA)-based PID. By incorporating PSO and IFOA into the PID-parameter searching processing, a globally optimal PID-parameter set can be found. Both numerical and experimental studies were conducted to evaluate the vibration isolation performance of the new controller. The results validated that the PSO-IFOA method can optimize the PID parameters for controlling the semi-active seat suspension and outperforms the other controllers.

For the application of vehicle suspensions, in the work by VivasLopez et al., multi-body dynamic analysis considering the mechanical components-nonlinear MR damper and the joints of a McPherson suspension was conducted. The stress concentration analysis was also given for the semi-active suspension performance evaluation. It provided very helpful results that the semi-active suspension will not only improve the vibration attenuation, but also decrease the stress concentrations in the suspension elements.

Dong et al. proposed a new variable stiffness and damping vibration absorber using MR technology for torsional vibration application - powertrain systems. Detailed model of the system input excitation from the powertrain system as well as the human-simulated intelligent controller for the new MR semiactive system are provided. With consideration of the nonlinear hysteresis of the components, reasonable results were provided in the work. The proposed concept of the smart device would be helpful for engineering applications.

Finally, we know that the selected topics and papers are not a comprehensive representation of the area of this special issue. Nonetheless, they represent the rich and many-faceted knowledge that we have the pleasure of sharing with the readers. We would like to express appreciation to the authors for their excellent contributions, to the reviewers for the quality check of the special 
section, and to the Frontiers in Materials Editors and the Editorial office staff for their great support.

\section{AUTHOR CONTRIBUTIONS}

X-XB: Writing - original draft, review, and editing RS: Writing original draft, review, and editing JG: Writing - original draft, review, and editing SS: Writing - original draft, review, and editing.
Conflict of Interest: The authors declare that the research was conducted in the absence of any commercial or financial relationships that could be construed as a potential conflict of interest.

Copyright $\odot 2021$ Bai, Sedaghati, Goldasz and Sun. This is an open-access article distributed under the terms of the Creative Commons Attribution License (CC BY). The use, distribution or reproduction in other forums is permitted, provided the original author(s) and the copyright owner(s) are credited and that the original publication in this journal is cited, in accordance with accepted academic practice. No use, distribution or reproduction is permitted which does not comply with these terms. 